\title{
Acute pulmonary hypertension in a patient with leptospirosis
}

\author{
PDPS Malalasekera ${ }^{1}$, MK Ragunathan ${ }^{2}$ \\ ${ }^{I}$ Registrar in Medicine, ${ }^{2}$ Consultant Physician, Teaching Hospital, Karapitiya, Galle.
}

\section{Introduction}

Leptospirosis is a zoonosis which is caused by leptospira species, mainly by Leptospira Interrogans. It shows a worldwide distribution mainly in tropics with exclusion of Polar Regions. Natural hosts are various mammals while man is accidentally infected after exposure to the environment contaminated with animal urine.

After an incubation period of 2 - 26 days, it may manifest as a self-limiting infection or a potentially fatal illness accompanied by multiorgan failure. The clinical course may be complicated by acute renal failure, uveitis, hemorrhage, ARDS, myocarditis, meningism and rhabdomyolysis.

We are reporting a clinical case which was complicated by reversible cardiomegaly and severe pulmonary hypertension. To our knowledge a case of this nature has not been reported in the local literature.

\section{Case Report}

A 58 year old previously healthy male from Galle was admitted with a history of fever, shortness of breath, chest pain at rest and diarrhoea of 3 day duration.

On admission, the patient was extremely ill and hemodynamically unstable with an elevated Jugular Venous Pressure. He was severely dyspnoeic and had crepitations over both lung fields.

ECG showed curved ST segment elevations in several leads. Arterial blood gas showed a type I respiratory failure. Chest X-ray revealed cardiomegaly and dilated pulmonary arteries suggestive of pulmonary hypertension (Fig 1).
Cardiac 2DEcho showed moderate to severe pulmonary hypertension with marked dilatation of the main pulmonary artery (diameter of 26 $\mathrm{mm})$.

Our presumptive differential diagnoses were Community Acquired Pneumonia with myocarditis and Leptospirosis complicated by pneumonitis and myocarditis.

The patient was started on IV Cefotaxime $1 \mathrm{~g}$ tds and IV Clarithromycin $500 \mathrm{mg}$ bd empirically to cover both infections.

Initial hematological investigations revealed a neutrophil leucocytosis with a platelet count of 20,000 with a PCV of $37 \%$. He had no bleeding manifestations. Urine full report showed red cells and granular casts. Though his renal and liver function tests remained normal, a high degree of clinical suspicion of leptospirosis was entertained.

As the patient's respiratory symptoms progressed with worsening of hemodynamic instability, he required ITU care with inotropic support and intubation. After 5 days of ITU care, the patient was transferred back to the ward and antibiotics were continued for a further one week.

Gradually his clinical condition improved. After full recovery a repeat chest X-ray and a 2DEcho was done to assess the improvement. Amazingly, the chest X-ray and the 2DEcho were completely normal. There was no evidence of pulmonary hypertension (Fig 2).

Ultimately clearing our doubts regarding the diagnosis, the leptospira antibody test was positive with a very significant titre of 3,200 . 


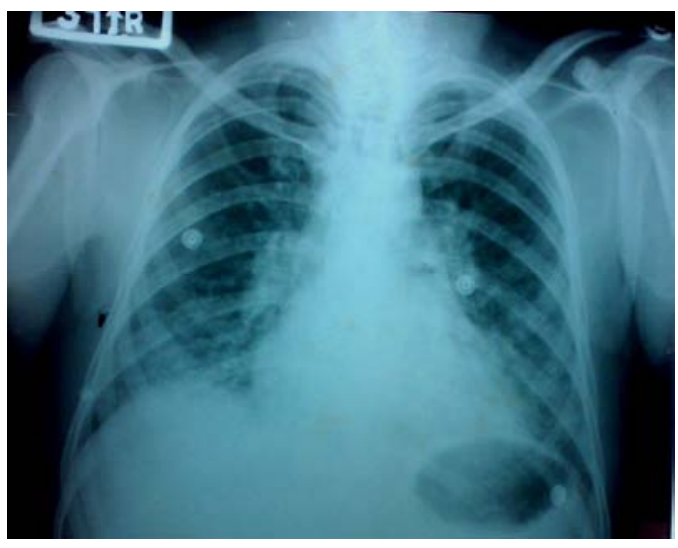

Figure 1 - X-Ray with cardiomegaly \& pulmonary hypertension

\section{Discussion}

As already discussed, leptospirosis is associated with a variable clinical course. Mainly it is an asymptomatic self-limiting infection. Sometimes it may manifest as a potentially fatal infection.

Abrupt onset of fever with rigors, myalgia and headache are the main presenting features in 75 $100 \%$ of patients.

Acute renal failure, haemorrhagic manifestations, adult respiratory distress syndrome, myocarditis, liver involvement and rhabdomyolysis are some of the well known complications of leptospirosis. Though known to occur, liver failure usually does not cause death. Vasculitis with necrosis of extremities may be seen in severe cases.

The potential severity of leptospirosis was illustrated in a retrospective study of 60 patients requiring ICU care in India ${ }^{1}$. The total mortality was found to be $52 \%$.

Severe pulmonary manifestations such as hemorrhage and ARDS are well recognized complications ${ }^{2}$. In another study in Peru among 321 patients with serological and clinical evidence of leptospirosis, severe pulmonary manifestations were seen in $3.7 \%$ of patients. Five out of seven patients died from pulmonary hemorrhage and ARDS.

Only one case was reported with reversible cardiomegaly with pulmonary hypertension in a study done in North America ${ }^{3}$. This has occurred after penicillin therapy and was

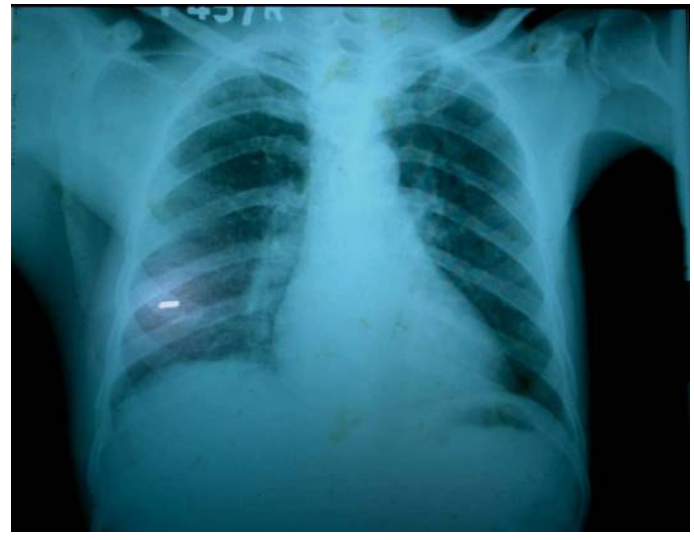

Figure 2 - Normal X-Ray after recovery

attributed to a Jarisch Herxheimer reaction to penicillin.

Leptospirosis is mainly a clinical diagnosis. Full blood count usually reveals a neutrophil leucocytosis with a low platelet count while urinalysis show proteinuria, pyuria, granular casts and microscopic hematuria. Elevated creatine kinase is found in $50 \%$ and may be useful as a diagnostic clue. Culture and PCR also assist in the diagnosis but is successful in only $50 \%$ of cases.

The gold standard serological test is considered to be microscopic agglutination test. It is most specific when a four fold or greater rise in titre is detected. In our clinical case, the titre was 3200 , which is very significant. The convalescent sample also showed the same titre. The titre was normal in the second sample taken one month after obtaining the first convalescent sample.

When we consider the therapy of leptospirosis, penicillins, tetracyclines, chloramphenicol and erythromycin have antileptospiral activity. Currently the widely used antibiotics are penicillin, third generation cephalosporins such as cefotaxime and ceftriaxone and doxycycline.

According to recent studies, the above antibiotics are therapeutically equivalent. Our patient was treated with IV cefotaxime.

The prophylactic use of doxycycline $200 \mathrm{mg} /$ week, 2-3 weeks prior to exposure and a $200 \mathrm{mg}$ stat dose after the exposure is a very important preventive method for this potentially fatal illness. 


\section{References}

1. Chawla V, Trivedi TH, Yeolekar ME. Epidemic of leptospirosis: an ICU experience. J Assoc Physicians India 2004; 52: 619.

2. Dolhnikoff Marisa, Mauad Thais, Bethlem Eduardo P, Carvalho Carlos RR. Leptospiral pneumonias. Current Opinion in Pulmonary Medicine 2007 May; 13(3): 230-5.
3. Emmanouilides CE, Kohn OF, Garibaldi R. Leptospirosis complicated by a Jarisch - Herxheimer reaction and adult respiratory distress syndrome: A case report. Clin Infect Dis 1994; 18: 1004. 